\title{
Fast and robust small infrared target detection using absolute directional mean difference algorithm
}

\author{
Saed Moradi, Payman Moallem*, Mohamad Farzan Sabahi \\ Department of Electrical Engineering, Faculty of Engineering, University of Isfahan, Isfahan, Iran
}

\begin{abstract}
Infrared small target detection in an infrared search and track (IRST) system is a challenging task. This situation becomes more complicated when high gray-intensity structural backgrounds appear in the field of view (FoV) of the infrared seeker. While the majority of the infrared small target detection algorithms neglect directional information, in this paper, a directional approach is presented to suppress structural backgrounds and develop a more effective detection algorithm. To this end, a similar concept to the average absolute gray difference (AAGD) is utilized to construct a novel directional small target detection algorithm called absolute directional mean difference (ADMD). Also, an efficient implementation procedure is presented for the proposed algorithm. The proposed algorithm effectively enhances the target area and eliminates background clutter. Simulation results on real infrared images prove the significant effectiveness of the proposed algorithm.
\end{abstract}

Keywords: small infrared target detection, directional mean difference, average absolute gray difference, structural background, real-time implementation

\section{Introduction}

Infrared imaging plays a vital role in a wide range of applications in remote sensing $[1,2]$ and long-distance target detection is of great importance for surveillance applications. In this filed, small infrared target detection remains a major challenge despite advances in infrared detector technology and image processing techniques [3]. This challenge becomes more critical when the long-distant target is embedded in a noisy and complicated background [4].

There are too many researches in the literature which are dedicated to the small infrared target detection field. The target detection procedure can be considered as a binary classification problem. Therefore, the detection process can be done using machine learning-based algorithms and different classifiers. Principal component analysis (PCA) [5], probabilistic principal component analysis (PPCA) [6, 7], robust principal component analysis (RPCA) [8], and nonlinear principal component analysis (NPCA) [9] were presented for small infrared target detection. Sparse representation-based methods detect small targets by decomposing each image patch over an over-complete dictionary. The over-complete dictionary can

\footnotetext{
*Corresponding author.

Email address: p_moallem@eng.ui.ac.ir (Payman Moallem)
} 
be constructed using Gaussian spatial distribution model for the small infrared target [10]. Also, the dictionary may be constructed according to background features [11]. Support vector machine (SVM) [12], and artificial neural networks [13] are also used as classifiers to distinguish small targets from background clutters. While the machine learning-based methods may work well in some situations, the overall detection ability and false-alarm rate drastically depend on the training samples and features.

Single-frame filtering-based methods are the most practical methods in the literature. These methods are divided into two categories. The first category is background subtraction. The algorithms belong to this category like as max-mean [14], max-median [14], and morphology opening [15] are fast and have low computational complexity. Although, these methods have a high false-alarm rate due to imperfect background estimation. The other type of single-frame filtering-based small target detection algorithms simultaneously enhances the target area and suppresses the background clutter. Laplacian of Gaussian (LoG) scalespace was proposed to distinguish the target from background clutter by maximum selection along scale dimension [16]. An extension to this method was presented in [17], where the morphological operators were utilized after LoG filtering to eliminate remaining background clutter. While the LoG operator is able to detect low-contrast point-wise targets, the second derivative operator boosts background noise. The difference of Gaussian (DoG) which is an efficient approximation of LoG was used in $[18,19]$ for detecting dim and small targets. Difference of Gabor (DoGb) and improved difference of Gabor (IDoGb) were presented in [20] to address false responses of the DoG filter along edges. The combination of DoG and frequency domain approach was presented in [21]. Local contrast measure (LCM) [22] has a good ability to enhance target region. However, LCM amplifies the single-pixel salt noise and increases the number of false-alarms. Additionally, the high-intensity background clutters are also intensified in the output image. Improved local contrast measure (ILCM) [23], novel local contrast measure (NLCM) [24], and relative local contrast measure (RLCM) [25] are the extended algorithms inspired by human visual system. Average absolute gray difference (AAGD) algorithm [26] effectively suppresses background noise (using local averaging) and enhances target area (by adopting local contrast). Since this algorithm can be easily implemented via local averaging and subtraction operators, the AAGD algorithm has low computational complexity and is suitable for real-time practical applications. However, AAGD faces a major problem when the infrared scenario contains high-intensity edges and structural background clutter. In this situation, AAGD returns false responses as true target areas. In multi-scale patch-based contrast measure (PCM) [27], after dividing each image patch into nine cells, the dissimilarities between the surrounding cells and the central one are calculated. By multiplying directional dissimilarities and minimum selection among the results, the final output is obtained. While this method utilizes the directional approach, however, by multiplying the directional dissimilarities, the final saliency map contains strong responses in non-target area. For instance, directional dissimilarities have high negative value for hole-like small objects. Thus, multiplying these negative values leads to high-intensity false responses. Note that, multiplying different directional information is the mainly used directional data combination in the literature [28, 29, 30, 31, 32].

The temporal information through successive frames can also be used to further falsealarms reduction [33]. Generally, in spatio-temporal (multi-frame) methods, the moving small infrared target is distinguished from random noise and cloud clutter using a predefined motion 
model [34]. However, there are two main challenges regarding utilizing multi-frame methods. First, the successive frames should be registered before processing, while the registration procedure is a challenging task for the textureless sky background. Second, the target with radial motion cannot be identified using this approach because the location of the target does not change over the successive frames.

In order to develop a robust and effective small target detection algorithm and address the aforementioned issues, in this paper, a directional approach is presented and a novel algorithm called absolute directional mean difference (ADMD) is proposed. The rest of this paper is organized as follows. In the next section, the related background and motivation is discussed briefly. The third section is dedicated to the proposed small target detection algorithm followed by simulation results in the fourth section. Finally, the paper is concluded in the fifth section.

\section{Background and motivation}

\subsection{Average absolute gray difference algorithm}

Average absolute gray difference (AAGD) is a recently presented algorithm for small infrared target detection in the literature [26]. Since this algorithm utilizes local averaging, it has good capability to eliminate background noise. The target enhancement mechanism in this method relies on the existing contrast between the target region and local background area. This algorithm is utilizing two nested windows which are slid pixel by pixel. The difference between the average values of the internal window $\Phi$ (Figure 1) and the external window $\Omega$ is used to construct a saliency map. Note that, the internal and external windows may be called target and background windows, respectively. The saliency map construction in AAGD algorithm can be mathematically formulated as

$$
A A G D=\left|\frac{1}{N_{\Phi}} \sum_{(s, t) \in \Phi} I(s, t)-\frac{1}{N_{\Omega}} \sum_{(p, q) \in \Omega} I(p, q)\right|^{2}
$$

where $I(x, y), N_{\Phi}$, and $N_{\Omega}$ stand for the pixel intensity at position $(x, y)$, the total number of pixels contained in the set $\Phi$, and the total number of pixels contained in the set $\Omega$, respectively.

AAGD algorithm has a good ability to enhance low contrast target and eliminate background noise. However, when the infrared scenario contains high-intensity edges and structural background clutter, non-target areas are also intensified. Also, while the target area always has positive contrast, AAGD returns strong responses no matter whether the contrast of an interested region is positive or negative [35].

To address the aforementioned issues and overcome deficiencies of the AAGD algorithm, here, a directional approach is utilized to achieve better clutter rejection and background suppression capability. The proposed approach is adopted in the AAGD algorithm and a new small infrared target detection algorithm called absolute directional mean difference (ADMD) is developed. The following section describes the proposed algorithm. 


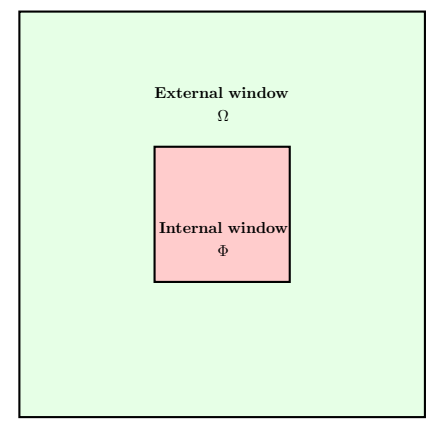

Figure 1: The internal as well as the external windows in the AAGD algorithm

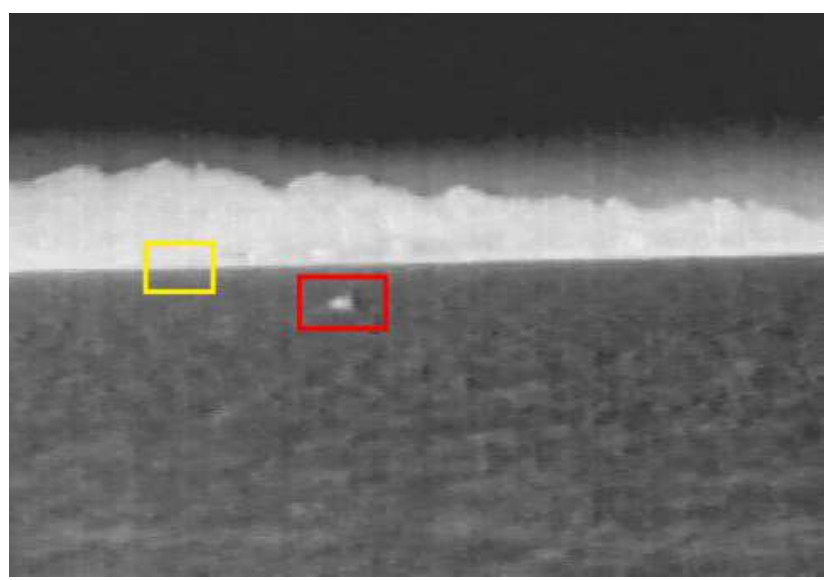

Figure 2: A target and a high-intensity edge in real infrared scenario

\section{The proposed algorithm}

While the directional filter design concept was presented and investigated in small infrared target detection field $[36,37,27,30]$, the lack of proper directional data combination strategy avoids robust small target detection. As mentioned in the introduction section, multiplication is the mainly used data combination strategy that has been investigated in the literature. Using multiplication to obtain final output from directional filters (similar to what proposed by $[36,37,27,30])$ will intensify the non-target areas. In order to address this issue and avoiding information loss, the combination strategy is modified in a way to improve clutter rejection ability.

Generally, infrared targets have positive local contrast which means that the target areas are brighter than the local background in all directions (neglecting special cases like the situation which the target is close to high-intensity background clutter). Considering this fact into account, it appears that utilizing all information from the all directional filters effectively can suppress high-intensity structural background. As shown in Figure 2, a small infrared target (surrounded by the red rectangle) has positive contrast in all directions, while a high-intensity edge (surrounded by the yellow rectangle) does not have such property. Hence, using the minimum value of the directional AAGD algorithm will effectively enhance falsealarm suppression ability. The following procedure describes the saliency map construction using the proposed method.

As depicted in Figure 3, two nested windows are assigned for each pixel $(i, j)$ in the input 


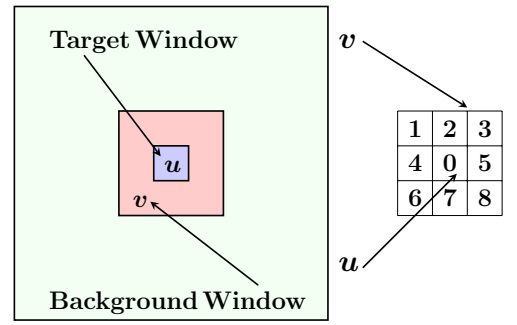

Figure 3: The target and background window construction in the proposed method

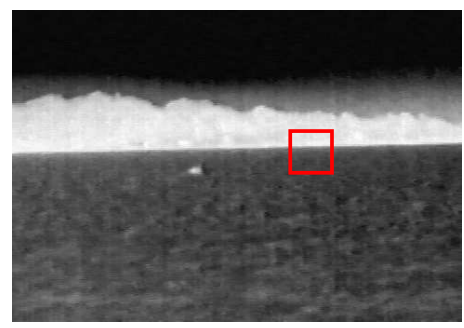

(a)

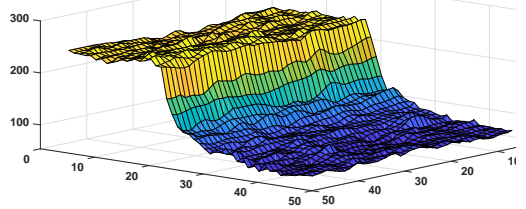

(d)

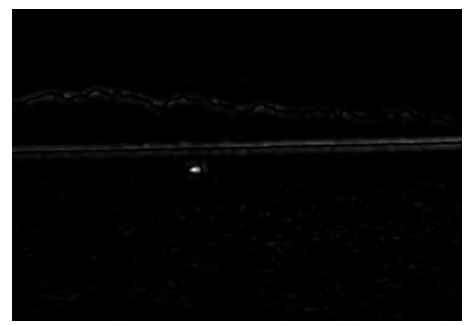

(b)

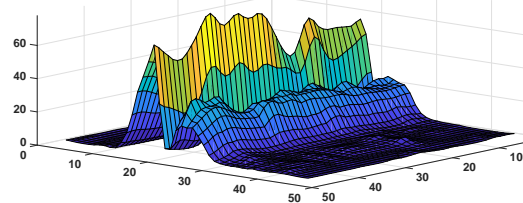

(e)

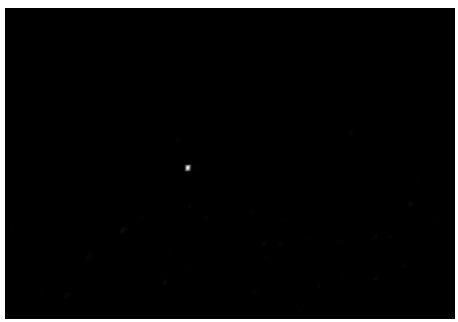

(c)

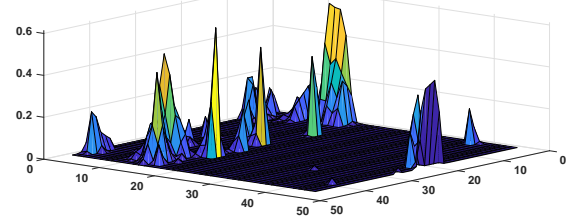

(f)

Figure 4: The output response of the AAGD as well as the proposed algorithm to a sharp edge: a) original input image, b) the filtering result of the AAGD algorithm, c) the filtering result of the proposed algorithm, d) 3-D surface of the red rectangle in the input image, e) 3-D surface of the red rectangle in the filtering result of the AAGD algorithm, f) 3-D surface of the red rectangle in the filtering result of the proposed algorithm. Note that, the gray intensity of all images are normalized to $[0-255]$ interval.

image. The internal one, $u$, denotes the target window and its size is related to the small infrared target size (typically smaller than $9 \times 9$ based on SPIE definition [38]). The external window, $v$, stands for local background window and its size is 3 times of the size of the target window. As shown in the figure, the external window is divided to 9 equal cells (obviously the $c e l l_{0}$ is the target window $u$ ). The average intensity of the pixels of the cell $k$ is denoted by $m_{k}$ and calculated as follows:

$$
m_{k}(i, j)=\frac{1}{N_{\text {cell }}} \sum_{(s, t) \in \text { cell }} I^{k}(s, t) \quad i=0,1, \ldots, 8
$$

where, $I^{k}(s, t)$ is the gray value of the pixel $(s, t)$ in $k^{t h}$ cell when the center of $c e l l_{0}$ lies on pixel $(i, j)$. Also, $N_{\text {cell }}$ is the number of pixels in each cell. The AAGD algorithm for eight different directions can be computed as:

$$
A A G D_{k}(i, j)=\left(m_{0}(i, j)-m_{k}(i, j)\right)^{2} \quad k=1, \ldots, 8
$$


In order to address the hole-like objects issue in the AAGD algorithm and reduce false alarms, simply the resulting negative contrast are suppressed using the following thresholding procedure:

$$
D_{k}(i, j)=A A G D_{k}(i, j) \times H\left(m_{0}(i, j)-m_{k}(i, j)\right) \quad k=1, \ldots, 8
$$

where $H(\cdot)$ is the Heaviside step function and expressed as:

$$
H(x)=\left\{\begin{array}{ll}
1 & x \geq 0 \\
0 & x<0
\end{array} .\right.
$$

As mentioned before, unlike the structural background clutters, small targets have positive contrast in all directions. Hence, considering minimum directional response as the final output will enhance clutter rejection capability, while the target detection ability remains unchanged. Therefore, in this paper, absolute directional mean difference (ADMD) value is defined as:

$$
A D M D(i, j)=\min \left\{D_{1}(i, j), D_{2}(i, j), \ldots, D_{8}(i, j)\right\}
$$

This procedure can be done through several scales (different cell size) for multi-scale processing. The output of the multi-scale ADMD algorithm can be expressed as:

$$
\begin{aligned}
& M S \_A D M D(i, j)= \\
& \max \left\{A D M D^{1}(i, j), A D M D^{2}(i, j), \ldots, A D M D^{S}(i, j)\right\}
\end{aligned}
$$

where $A D M D^{s}$ denotes the ADMD output at the $s^{\text {th }}$ scale.

\subsection{Clutter rejection mechanism}

Figure 4 shows the output response of the AAGD as well as the proposed algorithm to an infrared scene including a sharp cloud edge, which the gray intensity of all images are normalized to $[0-255]$ interval. As shown in the figure, the proposed algorithm effectively eliminates the high-intensity edge. The gray intensity of the red rectangle does not exceed 0.6 (Figure 4f). However, the AAGD algorithm has a relatively strong response in that area which is demonstrated as two parallel lines in the figure. As shown in Figure 4e, the maximum gray intensity of the area related to the red rectangle is about 60 which is approximately 100 times greater than the ADMD output.

In order to investigate the behavior of both AAGD and ADMD algorithms facing sharp edges, a brief analysis is provided here (Figure 5). Without loss of generality, let assume that the area under test just has two gray levels $\left(g_{b}\right.$ and $g_{d}$ values for bright and dark areas, respectively). Also, let assume that both target and background windows are identical for both algorithms. As the final assumption, let assume that the algorithms are constructed just in a single scale. In the first scenario (Figure 5a), the target window completely lies on 
bright area. In this situation, the output of the AAGD algorithm can be calculated as:

$$
\begin{aligned}
\text { out }_{A A G D} & =\left(g_{b}-\left(\frac{5 \times g_{b}+3 \times g_{d}}{8}\right)\right)^{2} \\
& =\left(\frac{3}{8}\left(g_{b}-g_{d}\right)\right)^{2}=\frac{9}{64} \Delta g^{2}
\end{aligned}
$$

According to Equation 8, as $\Delta g$ increases (the edge becomes more sharper), the output of the AAGD algorithm becomes more intensified (Figure 4e).

Considering Figure 5a as the input scenario, there are only two types of directional output in ADMD algorithm. $D_{b}$ for the directions along with bright background cells and $D_{d}$ for the directions along with dark background cells. These two outputs are calculated as follows:

$$
\begin{aligned}
& D_{b}=H\left(g_{b}-g_{b}\right) \times\left(g_{b}-g_{b}\right)^{2}=0 \\
& D_{d}=H\left(g_{b}-g_{d}\right) \times\left(g_{b}-g_{d}\right)^{2}=\Delta g^{2}
\end{aligned}
$$

Therefore,

$$
\text { out }_{A D M D}=\min \left\{D_{b}, D_{d}\right\}=\min \left\{0, \Delta g^{2}\right\}=0
$$

When the target window has negative contrast compared to the bright background cells (Figure 5b), the output of AAGD algorithm is computed as follows:

$$
\begin{aligned}
\text { out }_{A A G D} & =\left(g_{d}-\left(\frac{3 \times g_{b}+5 \times g_{d}}{8}\right)\right)^{2} \\
& =\left(\frac{3}{8}\left(g_{d}-g_{b}\right)\right)^{2}=\frac{9}{64} \Delta g^{2}
\end{aligned}
$$

Equation 11 demonstrates that, similar to the former scenario (Figure 5a), the AAGD algorithm returns a strong response for the non-target area. When the center of the target window exactly lies on the edge, the AAGD output takes zero value. This is why there is a dark area between two parallel lines in the AAGD output (Figure 4b).

The output of the ADMD algorithm to Figure 5b scenario is expressed as:

$$
\begin{aligned}
& D_{b}=H\left(g_{d}-g_{b}\right) \times\left(g_{d}-g_{b}\right)^{2}=0 \\
& D_{d}=H\left(g_{d}-g_{d}\right) \times\left(g_{d}-g_{d}\right)^{2}=0
\end{aligned}
$$

Therefore,

$$
\text { out }_{A D M D}=\min \left\{D_{b}, D_{d}\right\}=\min \{0,0\}=0
$$

According to Equation 10 and Equation 13, one can conclude that the proposed algorithm effectively eliminates high-intensity edges. It is worth mentioning that since the small target has positive contrast in all directions, minimum selection strategy does not degrade detection ability. 


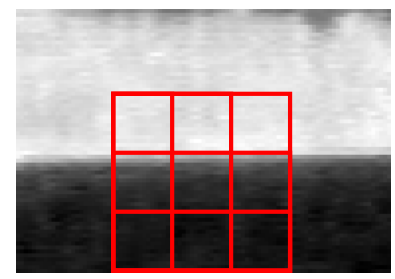

(b)

Figure 5: Two different scenarios to explain AAGD deficiency: a) the target window has positive contrast, b) the target window has negative contrast.

\subsection{Noise suppression ability}

At first sight, because of the smaller local background cell, it may seem that the proposed method has lower background noise rejection ability compared to the AAGD algorithm. However, the both minimum selection operator and negative value elimination strategy in the ADMD algorithm significantly improve the noise suppression ability of the proposed method. To compare the noise suppression ability of the AAGD as well as the proposed algorithm, 1-D additive white Gaussian noise (AWGN) is used as an input signal. The simulation is carried out 100000 times for the input noise signal and the mean and variance values of the output are taken into account. The mean and variance of the output noise response are shown in Figure 6. As shown in the figure, the proposed algorithm has better performance in background noise suppression.

In order to investigate how the proposed method copes with non-Gaussian noises, a similar simulation is carried out for Poisson as well as Rayleigh noises. As depicted in the Figure 7, the proposed method outperforms the AAGD, regardless of the noise distribution. Note that, regardless of the distribution of the background noise, the detection algorithm should be able to eliminate the noise and enhance only the true small target area. Thus, no prior assumption is made about the distribution of the background noise. However, modeling of background noise distribution is necessary for image restoration and noise removal, where the visual quality of the infrared scene is important. The interested reader may be referred to $[39,40,41]$ for further information.

\subsection{Efficient implementation}

In order to construct a single-scale ADMD algorithm, eight directional AAGD algorithms should be computed, separately. Performing this amount of operations needs high processing resources. In this section, the proposed algorithm is reformulated to reduce computational complexity.

Since structural background clutters do not have positive contrast in all directions, considering the maximum average intensity of the background cells in contrast function formulation will mimic the behavior of the proposed algorithm. Therefore, maximum directional mean value $M_{d i r}$ is defined as:

$$
M_{d i r}(i, j)=\max \left\{m_{1}(i, j), m_{2}(i, j), \ldots, m_{8}(i, j)\right\}
$$




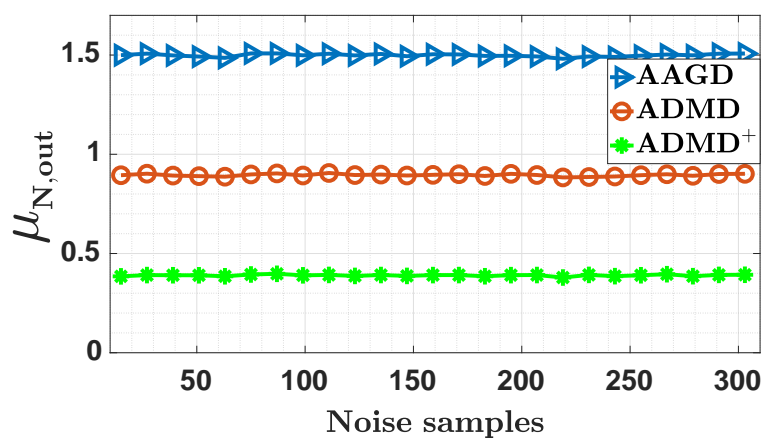

(a)

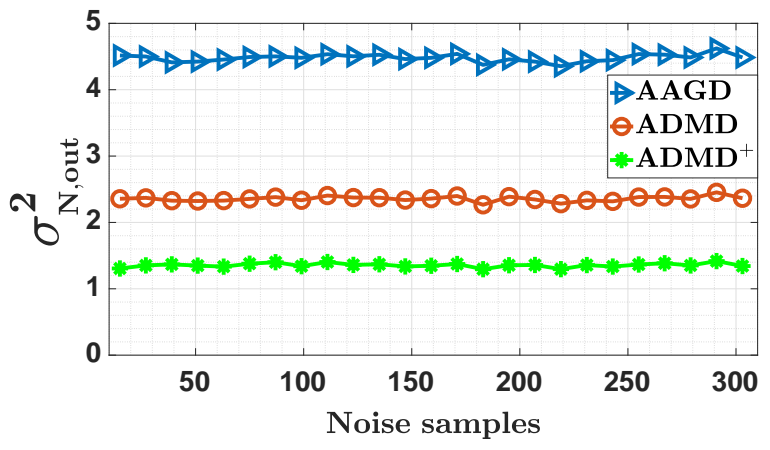

(b)

Figure 6: Output noise response for different algorithms (1-D cases): a) the mean value of output noise response, b) the variance of output noise response. ADMD and $\mathrm{ADMD}^{+}$denote the proposed algorithm without and with negative value elimination, respectively. The cell size (target window) and the size of AAGD background window are set to $9 \times 9$ and $27 \times 27$. Also, the standard deviation of the input noise $\left(\sigma_{N, i n}\right)$ is set to 3 .

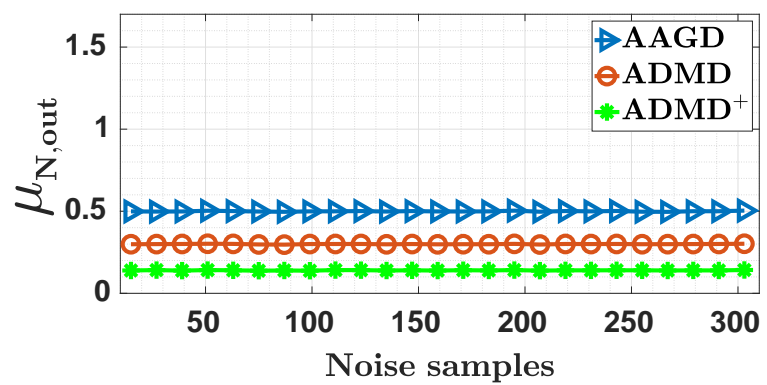

(a)

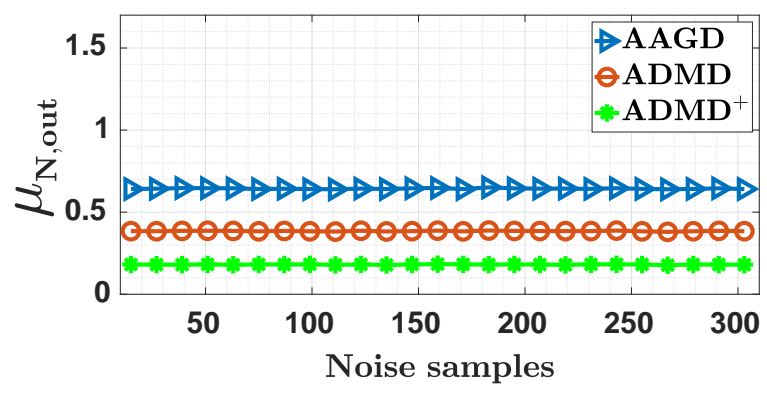

(c)

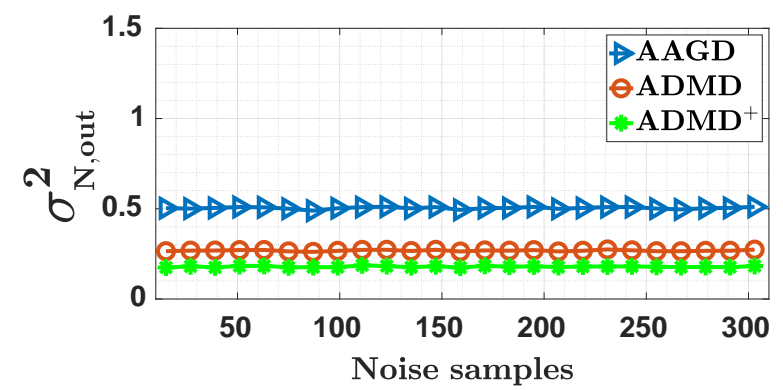

(b)

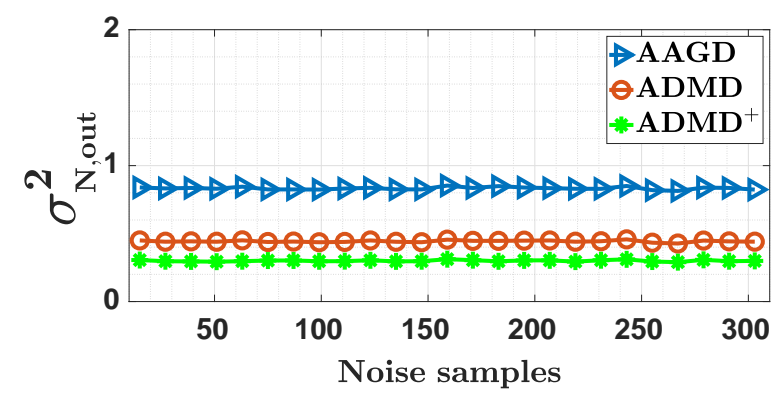

(d)

Figure 7: Output noise response for different algorithms (1-D cases): a) the mean value of output noise response when the input noise has Poisson distribution, b) the variance of output noise response when the input noise has Poisson distribution, c) the mean value of output noise response when the input noise has Rayleigh distribution, $\mathbf{d}$ ) the variance of output noise response when the input noise has Rayleigh distribution. The both $\lambda$ (for Poisson distribution) and scale parameter (for Rayleigh distribution) are set to 3 .

Accordingly, absolute difference mean value for the pixel $(i, j)$ is calculated as follows:

$$
A D M(i, j)=\left(m_{0}(i, j)-M_{\text {dir }}(i, j)\right)^{2}
$$

By discarding negative values through thresholding operation, the final ADMD output 


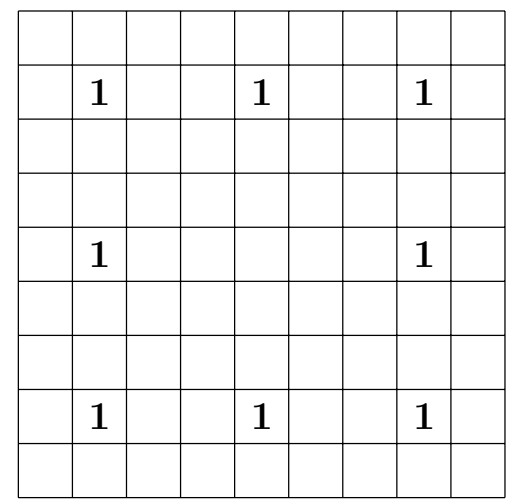

Figure 8: A $9 \times 9$ structural element to obtain maximum directional value for $3 \times 3$ target size (empty cells have zero values).

will achieve:

$$
A D M D(i, j)=A D M(i, j) \times H\left(m_{0}(i, j)-M_{\text {dir }}(i, j)\right)
$$

This new formulation can be efficiently implemented via local averaging and morphology dilation operators. To this end, the following procedure can be utilized to obtain the final saliency map:

1. The input infrared image is filtered using local averaging with proper neighborhood size $(3 \times 3,5 \times 5,7 \times 7$, or $9 \times 9), m_{0}$.

2. The filtered image $m_{0}$ is morphologically dilated using proper structural element (e.g. Figure 8) to obtain maximum directional value, $M_{d i r}$.

3. Then, the absolute difference mean is calculated, $A D M$.

4. Finally, the saliency map is constructed by eliminating negative contrasts, $A D M D$.

\section{Simulation results}

\subsection{Detection ability analysis}

In order to demonstrate the effectiveness of the proposed small target detection algorithm, simulation results are provided here. To compare detection performances, some basic as well

Table 1: SCR values for the test targets

\begin{tabular}{|c|c|c|c|c|c|c|}
\hline & AAGD & TopHat & LCM & PCM & LoG & ADMD \\
\hline Figure 9a & 4.37 & 1.94 & 1.55 & 3.78 & 2.43 & $\mathbf{8 6 . 2 3}$ \\
\hline Figure 9h & 9.07 & 7.17 & 1.78 & 3.01 & 7.27 & $\mathbf{2 2 . 8 5}$ \\
\hline Figure 9o & 17.31 & 10.62 & 2.32 & 17.14 & 12.56 & $\mathbf{2 2 . 6 5}$ \\
\hline Figure 9v & 11.59 & 3.14 & 2.29 & 8.09 & 5.25 & $\mathbf{1 1 . 9 6}$ \\
\hline Figure 9ac & 10.87 & 2.46 & 2.25 & 7.34 & 4.59 & $\mathbf{1 9 7 . 2 8}$ \\
\hline Figure 9aj & 13.69 & 5.22 & 1.66 & 6.23 & 6.95 & $\mathbf{5 4 9 . 1 8}$ \\
\hline
\end{tabular}




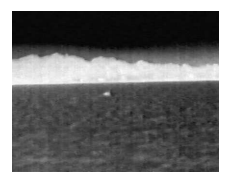

(a)

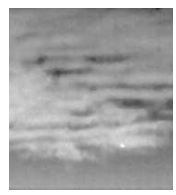

(h)

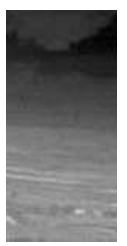

$(1$

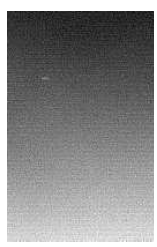

(v)

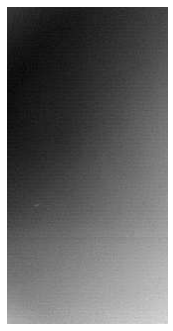

(ac)

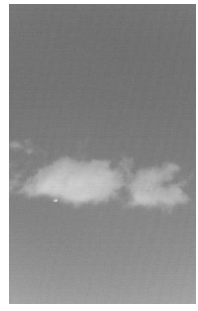

(aj)

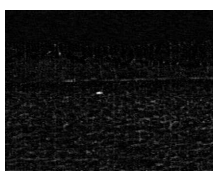

(b)

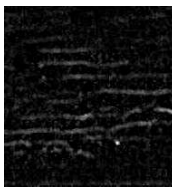

(i)
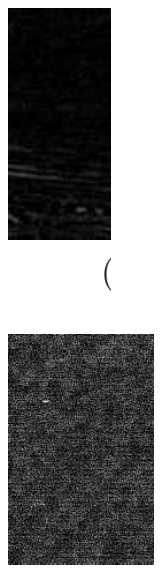

$(\mathrm{w})$

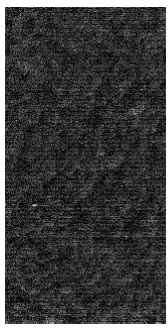

(ad)

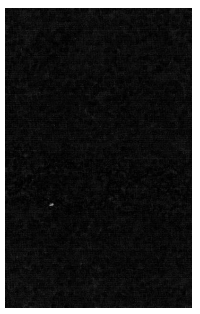

(ak)

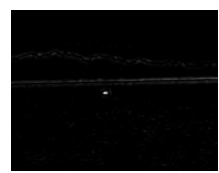

(c)

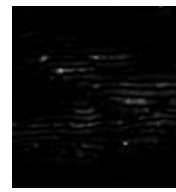

(j)
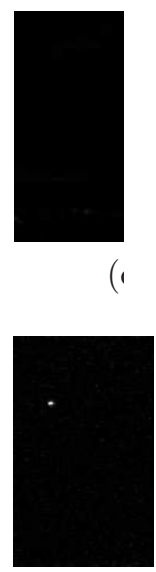

$(\mathrm{x})$

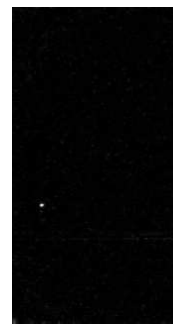

(ae)

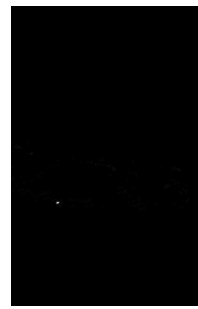

(al)

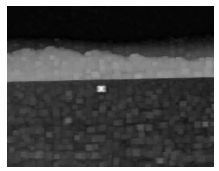

(d)

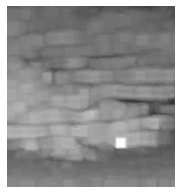

(k)
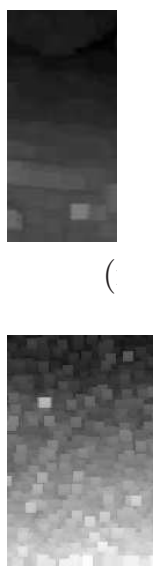

(y)

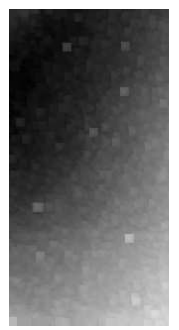

(af)

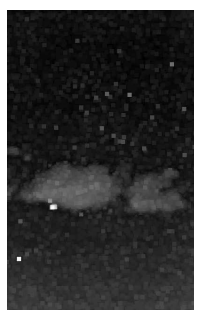

(am)

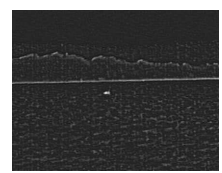

(e)

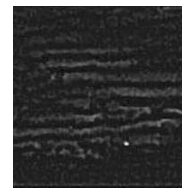

(1)
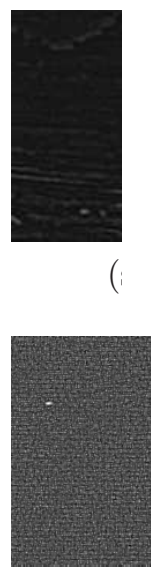

$(\mathrm{z})$

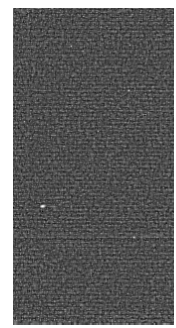

(ag)

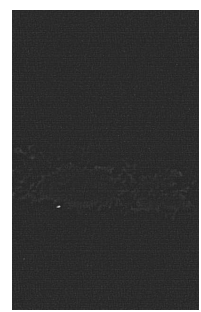

(an)

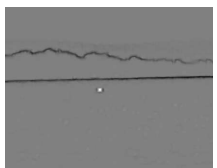

(f)

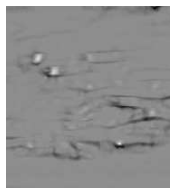

(m)
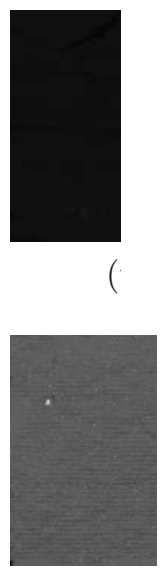

(aa)

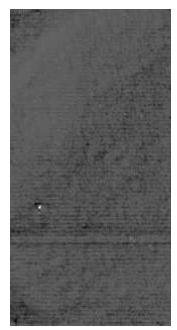

(ah)

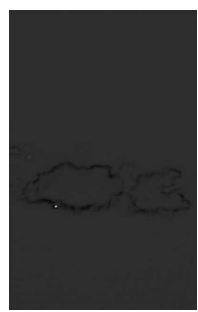

(ao)

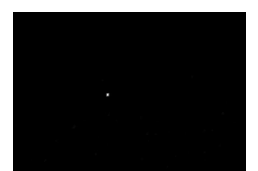

(g)

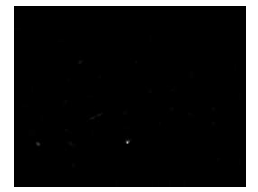

(n)

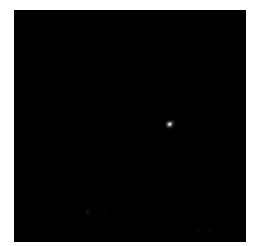

(u)

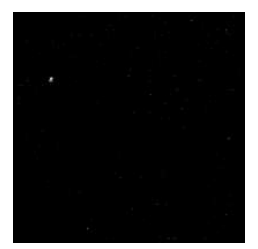

(ab)

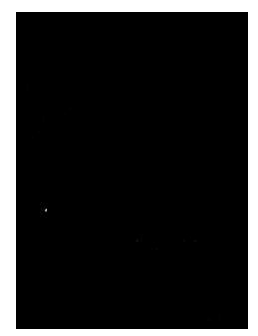

(ai)

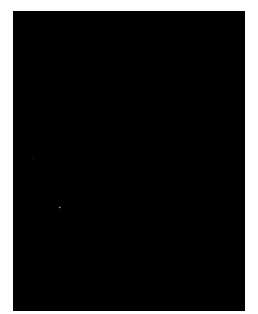

(ap)

Figure 9: pre-thresholding filtering results on the infrared images. $\mathbf{a}, \mathbf{h}, \mathbf{o}, \mathbf{v}, \mathbf{a c}, \mathbf{a j}$ ) original images, $\mathbf{b}, \mathbf{i}$, $\mathbf{p}, \mathbf{w}, \mathbf{a d}$, ak) filtering results of Top-Hat, $\mathbf{c}, \mathbf{j}, \mathbf{q}, \mathbf{x}, \mathbf{a e}$, al) filtering results of AAGD, d, k, r, y, af, am) filtering results of LCM, e, l, s, z, ag, an) filtering results of LoG, f, $\mathbf{m}, \mathbf{t}, \mathbf{a a}, \mathbf{a h}$, ao) filtering results of $\mathrm{PCM}, \mathrm{g}, \mathbf{n}, \mathbf{u}, \mathbf{a b}, \mathbf{a i}, \mathrm{ap})$ filtering results of the proposed algorithm (ADMD). 


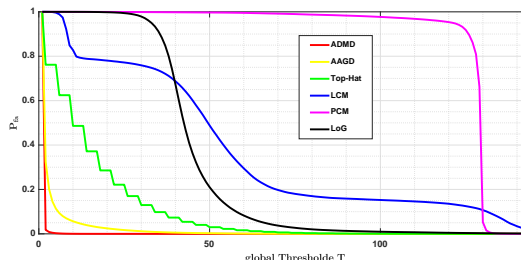

(a)

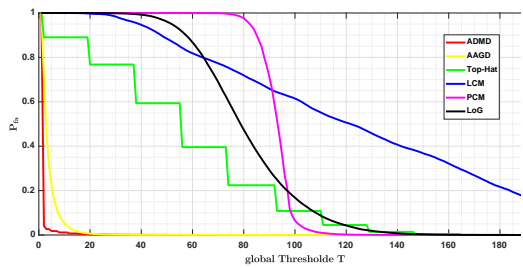

(d)

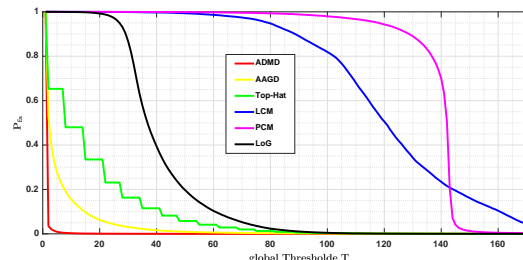

(b)

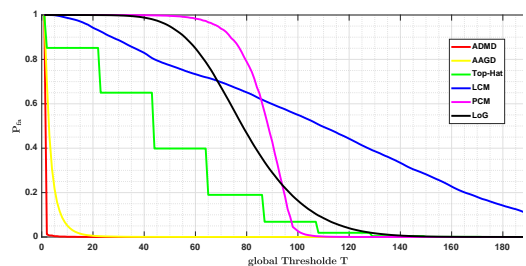

(e)

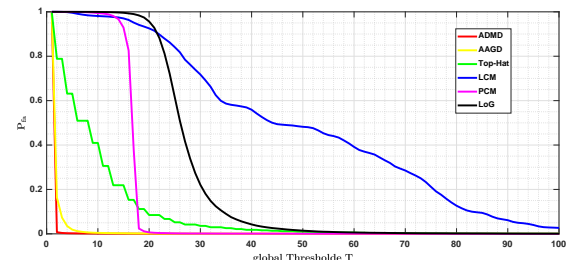

(c)

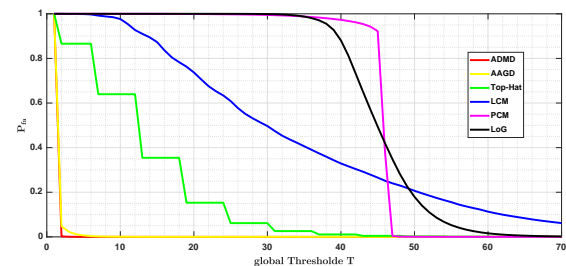

(f)

Figure 10: false alarm rate versus threshold level curves for the different test images including: a) Figure 9a, b) Figure 9h, c) Figure 9o, d) Figure 9v, e) Figure 9ac and f) Figure 9aj.

as state of the art algorithms like as Top-Hat [15], Laplacian of Gaussian (LoG) [42], local contrast measure (LCM) [22], absolute average gray difference (AAGD) [26], and patch-based contrast measure (PCM) [27] are chosen as baseline algorithms. Except for MS-LoG, which uses 12 scales[16], the rest of the algorithms (AAGD, LCM, PCM, and ADMD) utilize four scales. Typically, a small target occupies less than 80 pixels [38], therefore the target window in AAGD as well as the cell size in LCM and PCM take $[3 \times 3,5 \times 5,7 \times 7,9 \times 9]$ values to cover different size of the small target. The test images include naval and aerial infrared targets embedded in complicated backgrounds (Figure 9). The filtering results of the baseline algorithms as well as the proposed one are illustrated in Figure 9. As shown in the figure, the proposed algorithm (ADMD) effectively suppresses background clutter and enhances the small infrared target while the other methods show poor performances facing these scenarios. Note that, when the input image just contains homogeneous background plus detector noise (Figure 9v), since there is no structural texture, the performance of the AAGD algorithm is close to the proposed algorithm.

Background suppression factor (BSF) and signal to clutter ratio (SCR) which are defined

Table 2: BSF values for the test images

\begin{tabular}{|c|c|c|c|c|c|c|}
\hline & AAGD & TopHat & LCM & PCM & LoG & ADMD \\
\hline Figure 9a & 8.66 & 3.36 & 1.29 & 5.41 & 4.00 & $\mathbf{1 1 4 . 2 3}$ \\
\hline Figure 9h & 1.89 & 1.09 & 0.75 & 1.77 & 1.43 & $\mathbf{1 4 . 7 7}$ \\
\hline Figure 9o & 14.27 & 2.93 & 1.05 & 16.06 & 4.17 & $\mathbf{2 5 . 0 9}$ \\
\hline Figure 9v & 2.36 & 0.26 & 0.16 & 1.47 & 0.46 & $\mathbf{3 . 4 4}$ \\
\hline Figure 9ac & 4.88 & 0.55 & 0.29 & 1.81 & 0.85 & $\mathbf{2 5 . 0 1}$ \\
\hline Figure 9aj & 11.59 & 0.89 & 0.38 & 3.52 & 1.44 & $\mathbf{4 5 . 2 9}$ \\
\hline
\end{tabular}


as follows are used as quantitative measures:

$$
B S F=\frac{\sigma_{\text {in }}}{\sigma_{\text {out }}}, \quad S C R=\frac{f_{T}-f_{b}}{\sigma_{b}}
$$

where $f_{T}, f_{b}, \sigma_{b}, \sigma_{i n}$, and $\sigma_{\text {out }}$ denote average value of target pixels, mean of background region, standard deviation of local background, standard deviation of non-target area in original image, and standard deviation of non-target area in filtered image, respectively. A higher BSF value indicates better background clutter rejection and the more SCR the more possibility to hit the true target. These values are reported in Table 1 and Table 2 for the test images. As reported in the tables, it is clear that the ADMD algorithm strongly outperforms other methods from both SCR and BSF points of view.

While BSF and SCR are pre-thresholding metrics, false-alarm rate $\left(P_{f a}\right)$ is used to evaluate post-thresholding clutter rejection ability

$$
P_{f a}=\frac{N_{f}}{N_{w}}
$$

where $N_{f}$ and $N_{w}$ are the number of false alarms and number of all pixels in the whole image, respectively [43]. False-alarm rate versus global threshold level curves for the test images are shown in Figure 10. As depicted in the figure, the proposed algorithm has the lowest false-alarm rate compared to the other algorithms. This means that the proposed algorithm effectively suppresses background clutter.

\subsection{Execution time analysis}

In this section, the run-time analysis of the different algorithms is provided to demonstrate the computational complexity of each algorithm. All the algorithms are implemented using OpenCV C++ libraries. The full specifications of the implementation environment are reported in Table 3. Each detection algorithm is executed 100 times and the average execution time for the multi-scale (except MS-LoG algorithm which utilizes 12 scales [16], the other multi-scale algorithms consist of four identical scales) as well as the single-scale versions of the algorithms are reported in Table 4 and Table 5, respectively. As reported in the Table 4, the efficient implementation of the ADMD algorithm (ADMD eff) is almost 27 times faster than the original ADMD. Since the whole $288 \times 110000$ panorama image is captured every 1.5 seconds, none of these multi-scale algorithms can operate in real-time using the reported hardware (Table 3). GPU implementation will be a good choice for the real-time implementation of these algorithms. Table 5 shows the average execution time for single-scale algorithms. As reported in the table, TopHat, AAGD and ADMD eff $_{\text {algorithms }}$ can be implemented in real-time using the reported hardware. Also, it can be seen that the efficient $\mathrm{ADMD}_{\text {eff }}$ algorithm is significantly faster than the original ADMD algorithm.

\section{Conclusion}

Infrared small target detection is of great importance in a wide range of applications such as surveillance, remote sensing, security, and astronomy. Despite the high attention paid to this field, the lack of robust and effective target detection method is tangible. To develop a 
robust small target detection algorithm, in this paper, a directional approach is constructed for better background suppression. Since the small targets have positive local contrast in all directions, in the proposed method, after calculating the directional cell-based contrast for all eight directions, the minimum contrast is used as the output value. For a small target (which is an isotropic object in the image), all the directional contrasts have almost equal values. Thus, the minimum selection strategy does not affect the target enhancement ability. However, structural background shows different responses along the different direction. For instance, a sharp edge has zero contrast along the edge direction. Therefore, the minimum selection strategy will eliminate structural backgrounds. It may seem that the noise suppression ability decreases using the proposed approach because of that the local background cell in the proposed method is smaller than the local background window in AAGD algorithm. However, using the minimum selection strategy in ADMD suppresses background noise power much more compared to AAGD. Using SCR and BSF as pre-thresholding metrics shows that the ADMD algorithm outperforms the other baseline algorithms. Also, by constructing the false-alarm rate versus threshold values curve for the different algorithms, the advantage of the ADMD over AAGD algorithm is demonstrated in post-thresholding situation. Compared to the AAGD algorithm, the ADMD utilizes eight different directional contrasts which in turn increases the execution time of the ADMD algorithm. To address this problem, an efficient implementation of the ADMD algorithm is proposed through the reformulation of the algorithm. The improved implementation just utilizes an extra morphological dilation with a specific structural element. The improved implementation is almost 27 times faster than the original ADMD. All the proposed as well as the baseline algorithms are implemented using OpenCV C++ libraries. While the multi-scale version of the both AAGD and ADMD may not be implemented in real-time on old machines, the single-scale implementation of both algorithms is fast enough for practical applications.

\section{References}

[1] S. Türker-Kaya, C. W. Huck, A review of mid-infrared and near-infrared imaging: principles, concepts and applications in plant tissue analysis, Molecules 22 (1) (2017) 168.

[2] X. Jin, J. Li, T. J. Schmit, M. D. Goldberg, Evaluation of radiative transfer models in atmospheric profiling with broadband infrared radiance measurements, International journal of remote sensing 32 (3) (2011) 863-874.

Table 3: The full specifications of the implementation environment

\begin{tabular}{|c|c|}
\hline Operating System & Linux (Ubuntu 18.04, X64) \\
\hline Linux kernel version & $4.15 .0-42$-generic \\
\hline OpenCV version & 3.4 .3 \\
\hline Compiler & GCC 7.3 .0 \\
\hline Size of the test image & $288 \times 5600$ \\
\hline data depth and type & single channel 32 bit floating point \\
\hline CPU & Intel CORE i7-3520M @ 2.90GHz \\
\hline Memory & 8GB DDR3 @ $1600 \mathrm{MHz}$ \\
\hline
\end{tabular}




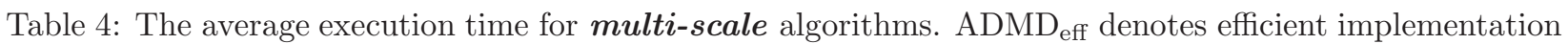
of the ADMD algorithm.

\begin{tabular}{|c|c|}
\hline Detection method & execution time $(\mathbf{m S})$ \\
\hline AAGD & 145.323 \\
\hline LCM & 4555.747 \\
\hline LoG & 213.667 \\
\hline PCM & 3463.643 \\
\hline ADMD & 4612.893 \\
\hline ADMD $_{\text {eff }}$ & 171.893 \\
\hline
\end{tabular}

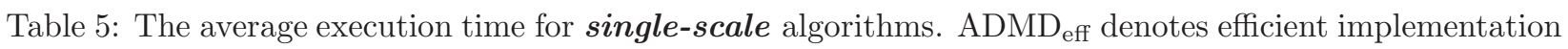
of the ADMD algorithm.

\begin{tabular}{|c|c|}
\hline Detection method & execution time $(\mathrm{mS})$ \\
\hline AAGD, $7 \times 7$ target window & 40.622 \\
\hline LCM, $7 \times 7$ cell size & 1111.990 \\
\hline TopHat, $7 \times 7$ flat SE & 4.536 \\
\hline PCM, $7 \times 7$ cell size & 1040.885 \\
\hline ADMD, $9 \times 9$ cell size & 1195.462 \\
\hline ADMD, $7 \times 7$ cell size & 1125.827 \\
\hline ADMD, $5 \times 5$ cell size & 1109.403 \\
\hline $\mathrm{ADMD}_{\text {eff }}, 9 \times 9$ cell size & 63.002 \\
\hline $\mathrm{ADMD}_{\text {eff }}, 7 \times 7$ cell size & 46.405 \\
\hline $\mathrm{ADMD}_{\text {eff }}, 5 \times 5$ cell size & 32.049 \\
\hline
\end{tabular}

[3] D. Liu, L. Cao, Z. Li, T. Liu, P. Che, Infrared small target detection based on flux density and direction diversity in gradient vector field, IEEE Journal of Selected Topics in Applied Earth Observations and Remote Sensing (99) (2018) 1-27.

[4] P. Khaledian, S. Moradi, E. Khaledian, A new method for detecting variable-size infrared targets, in: Sixth International Conference on Digital Image Processing, International Society for Optics and Photonics, 2014, pp. 91591J-91591J.

[5] T. Hu, J.-j. Zhao, Y. Cao, F.-L. Wang, J. Yang, Infrared small target detection based on saliency and principle component analysis, Journal of infrared and millimeter waves 29 (4) (2010) 303-306.

[6] C. Wang, S. Qin, Adaptive detection method of infrared small target based on targetbackground separation via robust principal component analysis, Infrared Physics \& Technology 69 (2015) 123-135.

[7] Y. Cao, R. M. Liu, J. Yang, Infrared small target detection using ppca, International Journal of Infrared and Millimeter Waves 29 (4) (2008) 385-395.

[8] Y. Dai, Y. Wu, Y. Song, Infrared small target and background separation via column- 
wise weighted robust principal component analysis, Infrared Physics \& Technology 77 (2016) 421-430.

[9] Z. Liu, C. Chen, X. Shen, X. Zou, Detection of small objects in image data based on the nonlinear principal component analysis neural network, Optical Engineering 44 (9) (2005) 093604.

[10] J. Zhao, Z. Tang, J. Yang, E. Liu, Infrared small target detection using sparse representation, Journal of Systems Engineering and Electronics 22 (6) (2011) 897-904.

[11] D. Liu, Z. Li, B. Liu, W. Chen, T. Liu, L. Cao, Infrared small target detection in heavy sky scene clutter based on sparse representation, Infrared physics \& technology 85 (2017) $13-31$.

[12] Y. Bi, X. Bai, T. Jin, S. Guo, Multiple feature analysis for infrared small target detection, IEEE Geoscience and Remote Sensing Letters 14 (8) (2017) 1333-1337.

[13] M. V. Shirvaikar, M. M. Trivedi, A neural network filter to detect small targets in high clutter backgrounds, IEEE Transactions on neural networks 6 (1) (1995) 252-257.

[14] S. D. Deshpande, M. H. Er, R. Venkateswarlu, P. Chan, Max-mean and max-median filters for detection of small targets, in: Signal and Data Processing of Small Targets 1999, Vol. 3809, International Society for Optics and Photonics, 1999, pp. 74-84.

[15] M. Zeng, J. Li, Z. Peng, The design of top-hat morphological filter and application to infrared target detection, Infrared Physics \& Technology 48 (1) (2006) 67-76.

[16] S. Kim, J. Lee, Scale invariant small target detection by optimizing signal-to-clutter ratio in heterogeneous background for infrared search and track, Pattern Recognition 45 (1) (2012) 393-406.

[17] X. Shao, H. Fan, G. Lu, J. Xu, An improved infrared dim and small target detection algorithm based on the contrast mechanism of human visual system, Infrared Physics \& Technology 55 (5) (2012) 403-408.

[18] X. Wang, G. Lv, L. Xu, Infrared dim target detection based on visual attention, Infrared Physics \& Technology 55 (6) (2012) 513-521.

[19] X. Dong, X. Huang, Y. Zheng, L. Shen, S. Bai, Infrared dim and small target detecting and tracking method inspired by human visual system, Infrared Physics \& Technology 62 (2014) 100-109.

[20] J. Han, Y. Ma, J. Huang, X. Mei, J. Ma, An infrared small target detecting algorithm based on human visual system, IEEE Geoscience and Remote Sensing Letters 13 (3) (2016) 452-456.

[21] X. Wang, Z. Peng, P. Zhang, Y. He, Infrared small target detection via nonnegativityconstrained variational mode decomposition, IEEE Geoscience and Remote Sensing Letters 14 (10) (2017) 1700-1704. 
[22] C. P. Chen, H. Li, Y. Wei, T. Xia, Y. Y. Tang, A local contrast method for small infrared target detection, IEEE Transactions on Geoscience and Remote Sensing 52 (1) (2014) $574-581$.

[23] J. Han, Y. Ma, B. Zhou, F. Fan, K. Liang, Y. Fang, A robust infrared small target detection algorithm based on human visual system, IEEE Geoscience and Remote Sensing Letters 11 (12) (2014) 2168-2172.

[24] Y. Qin, B. Li, Effective infrared small target detection utilizing a novel local contrast method, IEEE Geoscience and Remote Sensing Letters 13 (12) (2016) 1890-1894.

[25] J. Han, Y. Yu, K. Liang, H. Zhang, Infrared small-target detection under complex background based on subblock-level ratio-difference joint local contrast measure, Optical Engineering 57 (10) (2018) 103105.

[26] H. Deng, X. Sun, M. Liu, C. Ye, X. Zhou, Infrared small-target detection using multiscale gray difference weighted image entropy, IEEE Transactions on Aerospace and Electronic Systems 52 (1) (2016) 60-72.

[27] Y. Wei, X. You, H. Li, Multiscale patch-based contrast measure for small infrared target detection, Pattern Recognition 58 (2016) 216-226.

[28] S. Aghaziyarati, S. Moradi, H. Talebi, Small infrared target detection using absolute average difference weighted by cumulative directional derivatives, Infrared Physics \& Technology 101 (2019) 78-87.

[29] X. Zhang, Q. Ding, H. Luo, B. Hui, Z. Chang, J. Zhang, A directional-progressive search method for infrared small target detection, in: 2018 IEEE 3rd Optoelectronics Global Conference (OGC), IEEE, 2018, pp. 180-184.

[30] X. Zhang, Q. Ding, H. Luo, B. Hui, Z. Chang, J. Zhang, Infrared small target detection based on directional zero-crossing measure, Infrared Physics \& Technology 87 (2017) $113-123$.

[31] S. Qi, J. Ma, C. Tao, C. Yang, J. Tian, A robust directional saliency-based method for infrared small-target detection under various complex backgrounds, IEEE Geoscience and Remote Sensing Letters 10 (3) (2012) 495-499.

[32] C. Yang, J. Ma, M. Zhang, S. Zheng, X. Tian, Multiscale facet model for infrared small target detection, Infrared Physics \& Technology 67 (2014) 202-209.

[33] B. Zhao, S. Xiao, H. Lu, D. Wu, Spatial-temporal local contrast for moving point target detection in space-based infrared imaging system, Infrared Physics \& Technology 95 (2018) 53-60.

[34] Z. Chen, T. Deng, L. Gao, H. Zhou, S. Luo, A novel spatial-temporal detection method of dim infrared moving small target, Infrared Physics \& Technology 66 (2014) 84-96. 
[35] S. Moradi, P. Moallem, M. F. Sabahi, A false-alarm aware methodology to develop robust and efficient multi-scale infrared small target detection algorithm, Infrared Physics \& Technology 89 (2018) 387-397.

[36] S. Qi, J. Ma, C. Tao, C. Yang, J. Tian, A robust directional saliency-based method for infrared small-target detection under various complex backgrounds, IEEE Geoscience and Remote Sensing Letters 10 (3) (2013) 495-499.

[37] C. Yang, J. Ma, S. Qi, J. Tian, S. Zheng, X. Tian, Directional support value of gaussian transformation for infrared small target detection, Applied optics 54 (9) (2015) 22552265 .

[38] S. Zhang, F. Huang, B. Liu, H. Yu, Y. Chen, Infrared dim target detection method based on the fuzzy accurate updating symmetric adaptive resonance theory, Journal of Visual Communication and Image Representation 60 (2019) 180-191.

[39] Z. Gong, Z. Shen, K.-C. Toh, Image restoration with mixed or unknown noises, Multiscale Modeling \& Simulation 12 (2) (2014) 458-487.

[40] V. Stojanovic, N. Nedic, Identification of time-varying oe models in presence of nongaussian noise: Application to pneumatic servo drives, International Journal of Robust and Nonlinear Control 26 (18) (2016) 3974-3995.

[41] V. S. D. Prsic, Robust identification for fault detection in the presence of non-gaussian noises: application to hydraulic servo drives, Nonlinear Dynamics 100 (2020) 22992313.

[42] S. Kim, J.-H. Lee, Robust scale invariant target detection using the scale-space theory and optimization for irst, Pattern analysis and applications 14 (1) (2011) 57-66.

[43] S. Moradi, P. Moallem, M. F. Sabahi, Scale-space point spread function based framework to boost infrared target detection algorithms, Infrared Physics \& Technology 77 (2016) $27-34$. 